\title{
PREVISÕES CLIMÁTICAS SAZONAIS SOBRE O BRASIL: AVALIAÇÃO DO REGCM3 ANINHADO NO MODELO GLOBAL CPTEC/COLA
}

\author{
RUBINEI DORNELES MACHADO, ROSMERI PORFÍRIO DA ROCHA \\ Universidade de São Paulo (USP), Departamento de Ciências Atmosféricas \\ rmvip@usp.br, rosmerir@model.iag.usp.br
}

Recebido Setembro 2009 - Aceito Junho 2010

\begin{abstract}
RESUMO
Este trabalho avalia o desempenho de previsões sazonais do modelo climático regional RegCM3, aninhado ao modelo global CPTEC/COLA. As previsões com o RegCM3 utilizaram $60 \mathrm{~km}$ de resolução horizontal num domínio que inclui grande parte da América do Sul. As previsões do RegCM3 e CPTEC/COLA foram avaliadas utilizando as análises de chuva e temperatura do ar do Climate Prediction Center (CPC) e National Centers for Enviromental Prediction (NCEP), respectivamente. Entre maio de 2005 e julho de 2007, 27 previsões sazonais de chuva e temperatura do ar (exceto a temperatura do CPTEC/COLA, que possui 26 previsões) foram avaliadas em três regiões do Brasil: Nordeste (NDE), Sudeste (SDE) e Sul (SUL). As previsões do RegCM3 também foram comparadas com as climatologias das análises. De acordo com os índices estatísticos (bias, coeficiente de correlação, raiz quadrada do erro médio quadrático e coeficiente de eficiência), nas três regiões (NDE, SDE e SUL) a chuva sazonal prevista pelo RegCM3 é mais próxima da observada do que a prevista pelo CPTEC/COLA. Além disto, o RegCM3 também é melhor previsor da chuva sazonal do que da média das observações nas três regiões. Para temperatura, as previsões do RegCM3 são superiores às do CPTEC/COLA nas áreas NDE e SUL, enquanto o CPTEC/COLA é superior no SDE. Finalmente, as previsões de chuva e temperatura do RegCM3 são mais próximas das observações do que a climatologia observada. Estes resultados indicam o potencial de utilização do RegCM3 para previsão sazonal, que futuramente deverá ser explorado através de previsão por conjunto.
\end{abstract}

Palavras chave: previsão sazonal, modelo climático regional, validação objetiva

\begin{abstract}
SEASONAL CLIMATIC FORECAST OVER BRAZIL: EVALUATION OF THE REGCM3 MODEL NESTED TO THE CPTEC/COLA GLOBAL MODEL

This study presents an assessment of seasonal forecasts from the regional climate model RegCM3 nested to the CPTEC/COLA global model. The used RegCM3 version is $60 \mathrm{~km}$ horizontal resolution over an area that includes large part of South America. RegCM3 and CPTEC/COLA forecasts were evaluated using the rainfall and air temperature analyses from Climate Prediction Center (CPC) and National Centers for Environmental Prediction (NCEP), respectively. From May 2005 to July 2007, 27 seasonal forecast of rainfall and air temperature (except for air temperature from CPTEC/COLA with 26 forecasts) were evaluated over three regions of Brazil: Northeast (NDE), Southeast (SDE) and South (SUL). RegCM3 forecasts were also compared with the climatologic fields of the analyses. The statistical indexes (bias, correlation coefficient, root mean square error and efficiency coefficient) showed that over the three regions (NDE, SDE and SUL) the RegCM3 predicted seasonal rainfall is closer to the observations than the CPTEC/COLA. Moreover, the RegCM3 is a best predictor of the seasonal rainfall than the average of the observations during the period. For air temperature, the RegCM3 forecasts perform better than the CPTEC/COLA over NDE and SUL regions, while the CPTEC/COLA shows better performance ovver SDE. Finally, the RegCM3 seasonal air temperature and rainfall estimated are closer to the observations than the observed climatology. These results suggest a potential utility of RegCM3 to be used for seasonal forecast which will be explored in the future using ensemble forecast.
\end{abstract}

KeyWords: seasonal forecast, regional climate model, objective evaluation 


\section{INTRODUÇÃO}

A redução de perdas e aumento de lucros em diversas áreas sócio-econômicas como agricultura, hidrologia e pesca, muitas vezes depende de erros ou acertos de previsões climáticas sazonais. Do ponto de vista científico os Modelos de Circulação Geral da Atmosfera (MCGAs) e os Modelos Climáticos Regionais (MCRs; Dickinson et al. 1989, Giorgi e Marinucci, 1991) estão sendo desenvolvidos para fornecerem tais previsões.

Segundo Sen et al. (2004a,b) o interesse nos MCRs deve-se à possibilidade de utilizar parametrizações físicas mais apropriadas para mesoescala e também à maior resolução espacial que estes modelos podem utilizar. Tais características são de especial interesse nas regiões onde forçantes orográficas e litorâneas regulam a distribuição espacial de variáveis atmosféricas (chuva, temperatura do ar, direção e velocidade do vento, etc.), proporcionando melhor entendimento dos processos de interação nuvem-radiação, convecção cumulus e de interação entre solo-superfície-atmosfera, diminuindo assim erros que nos MCGAs são associados à baixa resolução espacial. Significativos esforços estão sendo empregados no desenvolvimento e melhoria da técnica de modelagem climática regional, incluindo além do desenvolvimento de novos modelos, o acoplamento com vários componentes que definem o clima, tais como, o oceano, hidrologia, solo-vegetação, química da atmosfera e aerossóis (Sen et al., 2004a).

$\mathrm{Na}$ América do Sul, Alves et al. (2000) estudaram a precipitação nas estações de verão e inverno, e mostraram que a distribuição espacial e o número de dias com chuva de intensidade fraca a moderada foram relativamente bem simulados pelo modelo regional ETA, que utilizou como condição inicial e de fronteira a re-análise do National Centers for Enviromental Prediction (NCEP; Kalnay et al., 1996). No modo de previsão, Chou et al. (2000) analisaram as previsões para o mês de agosto (seco) e novembro (chuvoso) sobre a América do Sul com o ETA aninhado no MCGACPTEC/COLA (Centro de Previsão de Tempo e Estudos Climáticos/Center for Ocean-Land-Atmosphere Studies; Bonatti, 1996). Neste estudo mostraram que a precipitação mensal prevista pelo ETA foi mais próxima da observada do que a previsão do CPTEC/COLA. Posteriormente, ao avaliar previsões com o ETA para 20022003, também aninhado no CPTEC/COLA, Chou et al. (2005) obtiveram que, em geral, o ETA reduz a super-estimativa de chuva média quadrimestral do CPTEC/COLA aproximando-se das observações. No entanto, segundo Chou et al. (2005) o ETA ainda mantém alguns erros do CPTEC/COLA, com maiores erros localizados geograficamente ao longo da costa norte e nordeste do Brasil e sobre os Andes. Estas previsões também revelaram que, geralmente, o ETA mostrou-se como melhor preditor da chuva sazonal do que a climatologia.
Focando o nordeste do Brasil, Sun et al. (2005) analisaram previsões entre Janeiro-Junho do Regional Spectral Model (RSM), aninhado no MCGA ECHAM4.5 (EuropeanHamburg Model version 4.5) e utilizando TSM (temperatura da superfície do mar) observada, para um período de 30 anos (1971-2000). Sun et al. (2005) destacaram que o RSM resolve melhor tanto o padrão espacial como a variabilidade inter-anual da chuva sobre o Nordeste do Brasil. Estas mesmas previsões foram avaliadas por Alves et al. (2005), os quais mostraram que para a quadra chuvosa (fevereiro a maio) o ECHAM4.5 apresentou erro médio seco, enquanto o RSM corrigiu as previsões do ECHAM4.5 na costa leste do Nordeste, prevendo valores de chuva mais próximos do observado.

Analisando previsões do MCGA COLA forçado com TSM observada, Misra (2006) discutiu que a previsão de chuva entre Fevereiro-Abril, sobre o nordeste do Brasil, depende da anomalia de TSM sobre o Atlântico tropical. As maiores falhas na previsão de chuva ocorrem quando as anomalias de TSM, sobre o Atlântico tropical norte, são grandes e não correlacionadas com os padrões de anomalia sobre o Oceano Pacífico leste.

Para América do Sul vários trabalhos (Fernandez et al., 2006a-b; Seth e Rojas, 2003; Cuadra e da Rocha, 2006; Seth et al., 2007; Pal et al., 2007) analisaram as condições climáticas mensais e sazonais, além da variabilidade inter-anual, simuladas pelo MCR RegCM3 (Regional Climate Model version 3; Pal et al., 2007) com condições inicial e de fronteira, fornecidas por re-análises (NCEP e ECMWF). Além disto, simulações contínuas de 20 anos, onde o RegCM3 foi aninhado no MCGA ECHAM, foram analisadas tanto por Seth et al. (2007), como por Rauscher et al. (2007). Para o Brasil central, a análise da chuva diária obtida do RegCM3 mostra que este modelo antecipa tanto o início, como o fim da estação chuvosa (Rauscher et al., 2007), e possui baixa habilidade em simular a variabilidade inter-anual na região das monções da América do Sul (Seth et al., 2007). No entanto, para o nordeste do Brasil ambas as características da chuva são melhores representadas pelo RegCM3, do que pelo ECHAM (Rauscher et al., 2007; Seth et al., 2007). Nestas simulações, Seth et al. (2007) mostraram que a variabilidade inter-anual, com foco na variabilidade associada ao El-NiñoOscilação Sul, foi corretamente capturada pelo RegCM3. Investigando o verão austral, da Rocha et al. (2009) mostraram que mesmo subestimando a chuva média sazonal, o RegCM3 simulou de acordo com as observações os principais aspectos do ciclo diurno da chuva sobre a América do Sul. Maiores semelhanças foram encontradas sobre os trópicos e subtrópicos, que apresentam máximo de chuva à tarde (1500-1800 UTC) e mínimo pela manhã (0900-1200 UTC).

Para o Brasil, até o momento não se conhece o desempenho do RegCM3 para previsão sazonal, onde as 
condições iniciais mudam a cada mês e são fornecidas por previsões de MCGA. Portanto, o objetivo deste trabalho é avaliar as previsões sazonais do RegCM3 aninhado ao MCGA CPTEC/COLA, utilizando TSM persistida, ou seja, no modo de previsão. O período de análise se estende de maio de 2005 até agosto de 2007, o que fornece um total de 27 previsões, com o RegCM3 iniciado e dirigido com previsões do CPTEC/COLA.

Este artigo está organizado da seguinte maneira: no item 2 descreve-se os modelos RegCM3 e CPTEC/COLA, os dados utilizados e a metodologia para as previsões numéricas e validações; e no item 3 avalia-se o desempenho das previsões do RegCM3 e do CPTEC/COLA e também compara-se as previsões do RegCM3 com a climatologia observada. Por fim, o item 4 traz um sumário e conclusões deste trabalho.

\section{METODOLOGIA}

\subsection{Descrição do Modelo Climático Regional - RegCM3}

O modelo RegCM3 está descrito em Pal et al. (2007) e é um código de livre acesso à pesquisa, desenvolvido e distribuído pelo International Center for Theoretical Physics (ICTP). Este é um modelo de equações primitivas, de área limitada e em pontos de grade, com coordenada vertical sigma-pressão. O RegCM3 parametriza os processos físicos de interação solo-superfícieatmosfera, camada limite planetária (CLP), radiativos e também os processos úmidos. A interação solo-planta-atmosfera segue o Biosphere-Atmosphere Transfer Scheme (BATS; DICKINSON et al. 1993) e na CLP os fluxos verticais de calor, umidade e momento são calculados pela teoria $\mathrm{K}$ com a inclusão de um termo não local (Holstlag et al., 1990). Para a transferência radiativa o RegCM3 utiliza o mesmo esquema do CCM3 (Community Climate Model 3; Kiehl et al., 1996).

Nas previsões realizadas o RegCM3 utilizou a parametrização de convecção em cumulus profundo de Grell (1993) com o fechamento de Fritsch-Chappell (Fritsch e Chappell, 1980) e o esquema proposto por Pal et al. (2000) para resolver a precipitação na escala da grade.

\subsection{Condições iniciais e de fronteiras}

As condições iniciais e de fronteira para as previsões do RegCM3 foram fornecidas pelo MCGA CPTEC/COLA. A versão deste modelo utilizada neste trabalho é aquela descrita em Bonatti (1996), cuja resolução é T62L28, indicando truncamento triangular na onda zonal de número 62 e 28 camadas na vertical. Ainda segundo Bonatti (1996), o CPTEC/ COLA resolve as equações primitivas em coordenadas esféricas e na forma espectral. Para facilitar o tratamento espectral, as equações do movimento horizontal são transformadas nas equações da vorticidade e divergência. Portanto, as variáveis previstas pelo CPTEC/COLA são: logaritmo da pressão à superfície, vorticidade, divergência do vento horizontal, temperatura virtual e umidade específica, além das obtidas das parametrizações físicas (temperatura de superfície, do interior do solo e do interior do dossel, umidade do solo, chuva, etc.). O CPTEC/COLA parametriza os processos de interação solosuperfície-atmosfera, CLP, transferência radiativa na atmosfera, condensação de grande escala e convecção cumulus profundo e convecção rasa, além da difusão horizontal e local.

No modo de previsão climática, a anomalia de TSM observada nos primeiros 15 dias do mês inicial da previsão é persistida nos 4 meses seguintes, totalizando 4,5 meses de previsão do MCGA CPTEC/COLA. Após pós-processamento, as variáveis previstas pelo CPTEC/COLA foram convertidas em altura geopotencial, temperatura do ar, vento horizontal (componente zonal e meridional), umidade relativa e pressão ao nível médio do mar. Estas variáveis estão disponíveis a cada 6 horas e constituem a condição inicial e de fronteira para as previsões do RegCM3.

Os arquivos globais de topografia e cobertura vegetal, com resolução horizontal de 10', utilizados nas previsões do RegCM3 são os do United States Geological Survey (USGS) e Global Land Cover Characterization (GLCC) e estão descritos em Loveland et al. (2000).

O RegCM3 (aninhado ao CPTEC/COLA) foi integrado com 18 níveis sigma na vertical e $60 \mathrm{~km}$ de resolução horizontal no domínio apresentado na Figura 1. As previsões foram iniciadas sempre às 00 UTC do dia 16 de cada mês, e se estenderam até o último dia do terceiro mês subseqüente, totalizando 106 (ou mais) dias de previsão. Os primeiros 14 (ou 15, depende do número de dias do mês) de previsão, assim como em Misra e Kanamitsu (2004), foram considerados como de "spin-up" e excluídos das avaliações. Por exemplo, para uma previsão iniciada em 16 de maio o trimestre de avaliação corresponde a Junho-Julho-Agosto (JJA), enquanto uma previsão iniciada em janeiro o trimestre de avaliação é Fevereiro-Março-Abril (FMA), e assim sucessivamente. Neste trabalho são avaliadas 27 previsões, com a primeira iniciada em maio de 2005, trimestre de avaliação JJA/2005, e a última em julho de 2007, trimestre de avaliação ASO/2007.

\subsection{Dados para avaliação}

A análise de chuva diária do Climate Prediction Center (CPC; Silva et al., 2007), com resolução horizontal de $1^{\circ} \mathrm{x}$ $1^{\circ}$ de latitude por longitude, foi utilizada para avaliar a chuva média trimestral prevista pelos modelos (RegCM3 e CPTEC/ COLA). Enquanto a temperatura do ar (a $2 \mathrm{~m}$ de altura) média trimestral foi avaliada contra a da re-análise do NCEP (Kalnay et 
al. 1996), com resolução de $2,5^{\circ} \times 2,5^{\circ}$ de longitude por latitude. As climatologias observadas de precipitação (1978 a 2004) e de temperatura do ar (1950 a 1999) foram obtidas, respectivamente, da média trimestral do CPC e da re-análise do NCEP.

Os dados do CPC utilizam apenas chuva diária de pluviômetros para através da interpolação com o esquema de Cresmann obter a análise em pontos de grade. Para períodos mais recentes, a análise do CPC incluiu um grande número de pluviômetros, principalmente sobre o centro-leste e sul do Brasil (ver Figura 1 de Silva et al., 2007). Esta análise em pontos de grade pode ser utilizada em estudos hidrológicos, de variabilidade climática (Silva et al., 2007) e também para validação de simulações climáticas (da Rocha et al., 2009). Comparações da análise do CPC com observações da estação do IAG-USP na cidade de São Paulo, que possui observações confiáveis, mostram correlação superior a 0,72 para chuva mensal e diferença percentual da ordem de $10 \%$ nos valores sazonais (Figuras e Tabelas não mostradas). A re-análise do NCEP é amplamente utilizada em estudos climáticos e de variabilidade do clima. Embora a qualidade da chuva do NCEP seja questionável (ver da Rocha et al., 2009) a temperatura do ar tem correlação alta (superior a 0,7 ) com as observações da estação do IAG-USP. Adicionalmente, é a única análise de temperatura do ar disponível para períodos mais recentes, portanto, para ser utilizada no presente estudo.

\subsection{Metodologia da avaliação das previsões}

As previsões foram avaliadas através de análises subjetivas e objetivas dos campos médios trimestrais. Para avaliação objetiva, nas três regiões apresentadas na Figura 1 (denominadas de Nordeste, NDE, Sudeste, SDE, e Sul, SUL, do Brasil) calcularam-se médias trimestrais, coeficiente de correlação linear, coeficiente de eficiência de Nash e Sutcliffe (1970), com e sem a remoção do erro médio (ou bias), e a raiz quadrada do erro médio quadrático (rmse).

O coeficiente de eficiência (E) é definido em Nash e Sutcliffe (1970) como:

$$
E=1-\frac{\sum_{i=1}^{N}\left(P_{o}-P_{e}\right)^{2}}{\sum_{i=1}^{N}\left(P_{o}-\overline{P_{o}}\right)^{2}}
$$

onde $\mathrm{P}_{\mathrm{o}}$ e $\mathrm{P}_{\mathrm{e}}$ são os valores médios trimestrais observados e previstos, respectivamente, $\mathrm{N}$ o número de previsões e a barra superior indica o valor médio de $\mathrm{N}$ observações. Este coeficiente varia de menos infinito a 1 , sendo que o valor 1 indica perfeito ajuste entre previsões e observações, i.e., o coeficiente $\mathrm{E}$ indica o quão eficiente é a previsão comparada com o valor médio observado no período de validação. $\mathrm{O}$ coeficiente $\mathrm{E}$ fornecido por (1), onde as diferenças aparecem ao quadrado, ressalta a habilidade do modelo na previsão de extremos.

Segundo Weber et al. (1982), o uso de medidas de erro isoladamente pode ser uma forma inadequada de estimar o desempenho dos modelos, enquanto o emprego de várias medidas de erro em conjunto costuma ser um método mais coerente. Seguindo estas idéias, Silva (2008) discute que um conjunto de medidas também atua como um efeito compensatório, uma vez que cada índice estatístico possui uma lacuna que pode ser preenchida com o de outro. Sendo assim, para comparar as previsões do CPTEC/COLA e RegCM3 e estabelecer se previsões do modelo regional aprimoram ou não as do global, foram atribuídos escores

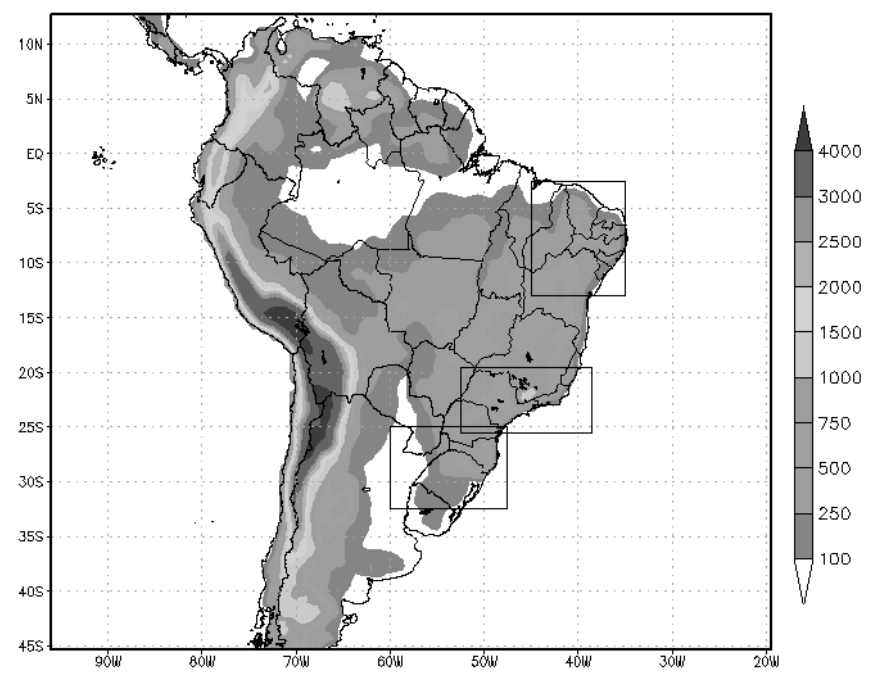

Figura 1 - Domínio e topografia (m) para previsões sazonais. Em destaque os três subdomínios (NDE, SDE e SUL) utilizados na avaliação das previsões. 
a cada índice estatístico, conforme definidos na Tabela 1. Índices estatísticos absolutos recebem escore com peso 1, enquanto os relativos recebem escore com peso 2. Os escores são atribuídos aos índices durante a comparação entre os dois modelos. Isto é feito de forma cumulativa, ou seja, se um determinado modelo obtém melhores valores em quatro índices, sua pontuação total é a soma dos escores atribuídos a cada um desses índices. É importante lembrar que quando os índices estatísticos são muito próximos entre si ambos os modelos pontuam.

As previsões do RegCM3 também foram comparadas com a climatologia observada através do coeficiente de eficiência E' (Nash e Suticliffe, 1970) definido como:

$$
E^{\prime}=1-\frac{\sum\left|P_{o}-P_{e}\right|}{\sum\left|P_{o}-\overline{P_{o}^{\prime}}\right|}
$$

onde $\overline{P_{o}^{\prime}}$ representa o valor climatológico trimestral observado. E', fornecido por (2), possui interpretação semelhante à E, i.e., quanto mais próximo de 1 maior é a indicação de que o modelo é melhor preditor do que a média, no caso a climatológica. Entretanto, se E'< 0 isto indicaria que o modelo é pior previsor do que a climatologia.

\section{RESULTADOS E DISCUSSÃO}

Como descrito, as previsões sazonais com o RegCM3 foram obtidas utilizando previsões do CPTEC/COLA como condições inicial e de fronteira. Portanto, é fundamental comparar ambas buscando identificar erros sistemáticos.

\subsection{Avaliações subjetivas}

A Figura 2 apresenta as análises do CPC e as previsões (CPTEC/COLA e RegCM3) para a precipitação média nos trimestres JJA/2005 (inverno austral) e DJF de 2005/2006 (verão austral). Para o inverno (Figura 2a), as maiores taxas de precipitação são observadas na parte central da região sul do Brasil, extremo norte da Amazônia e em uma faixa estreita no leste do nordeste do Brasil (desde o sul do Rio Grande do Norte até o sul da Bahia. Esta distribuição da chuva é coerente com o ciclo anual da atividade convectiva nestas regiões (Horel et al., 1989), onde o deslocamento da ZCIT (Zona de Convergência
Intertropical) para norte reduz a chuva no centro-norte do Brasil (Marengo et al. 2003), e na costa leste do nordeste a chuva resultaria da convergência dos alíseos de sudeste e a brisa terrestre (Kousky, 1980). O máximo de precipitação no sul do Brasil (Figura. 2a) resulta da atuação de sistemas frontais e ciclogêneses (Reboita et al., 2005). A previsão do RegCM3 (Figura 2b) localiza corretamente estas três áreas com precipitação, assim como a área mais seca na parte central do Brasil. Entretanto, o RegCM3 subestima (superestima) o máximo (mínimo) de chuva no sul do Brasil (centro-leste da Argentina). A subestimativa de chuva no sul pode estar associada à menor convergência de massa em $850 \mathrm{hPa}$, já que o vento de norte/noroeste que cruza o Paraguai e sul do Brasil neste nível é mais fraco no RegCM3 e CPTEC/COLA, do que no NCEP (Figuras não mostradas). Na previsão do CPTEC/COLA(Figura 2c) a chuva na região norte está deslocada para sul e há um prolongamento desta banda de precipitação até o nordeste do Brasil, o que não é observado no CPC (Figura 2a). Esta banda de chuva, desde a Amazônia até o nordeste, mostra-se como um erro sistemático do CPTEC/COLA, também é apresentado em Misra (2006) e nas previsões de JJA de 2006 e 2007 (Figuras não mostradas). Na análise do CPC, em JJA a ZCIT encontra-se no Hemisfério Norte, e aparentemente o CPTEC/COLA ainda mantém grande parte da atividade convectiva associada à ZCIT na parte central da Amazônia (Figura 2c). Comparativamente, as Figuras 2a-c indicam que o RegCM3 corrige, pelo menos em parte, o erro de posicionamento da ZCIT do CPTEC/COLA (Figura 2c). A larga faixa de chuva ao longo da costa do norte e nordeste do Brasil prevista pelo CPTEC/COLA (Figura 2c), em desacordo com a análise do CPC (Figura 2a), poderia resultar de deficiências na previsão da circulação em baixos níveis. Para JJA, os ventos de sudeste/leste em $850 \mathrm{hPa}$ no CPTEC/COLA são mais perpendiculares à costa, o que implicaria em maior convergência quando adentram o continente, do que no NCEP e RegCM3 (Figuras não mostradas). De maneira geral, aspectos regionais da chuva de JJA/2005 (máximo no sul, posicionamento da chuva no norte/nordeste do Brasil) previstos pelo RegCM3 (Figura 2b) são mais semelhantes à análise do CPC (Figura 2a) do que os obtidos do CPTEC/COLA (Figura 2c).

$\mathrm{Na}$ climatologia para o trimestre DJF as chuvas mais intensas situam-se no setor central e sul da região norte e centro-

Tabela 1- Escore dos índices estatísticos utilizados na avaliação das previsões.

\begin{tabular}{|c|c|c|}
\hline Símbolo (unidade) & Índices estatísticos & Escores \\
\hline $\mathrm{B}\left(\mathrm{mm} / \mathrm{dia}\right.$ ou $\left.{ }^{\circ} \mathrm{C}\right)$ & Erro médio (bias) & 1 \\
\hline $\mathrm{rmse}\left(\mathrm{mm} / \mathrm{dia}\right.$ ou $\left.{ }^{\circ} \mathrm{C}\right)$ & Raiz quadrada do erro quadrático médio & 1 \\
\hline $\mathrm{r}$ (admensional) & Correlação linear & 2 \\
\hline E (admensional) & Coeficiente de Eficiência & 2 \\
\hline $\mathrm{E}_{\mathrm{b}}$ (admensional) & Coeficiente de eficiência após remover o bias & 2 \\
\hline
\end{tabular}


oeste do Brasil, estendendo-se até a parte central da região sudeste, caracterizando assim a Zona de Convergência do Atlântico Sul - ZCAS (Kodama, 1992; Liebmann et al., 1999; Marengo et al.,

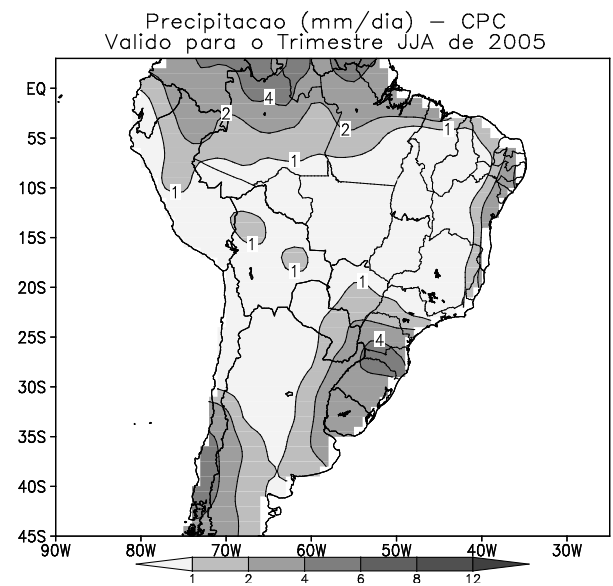

a)

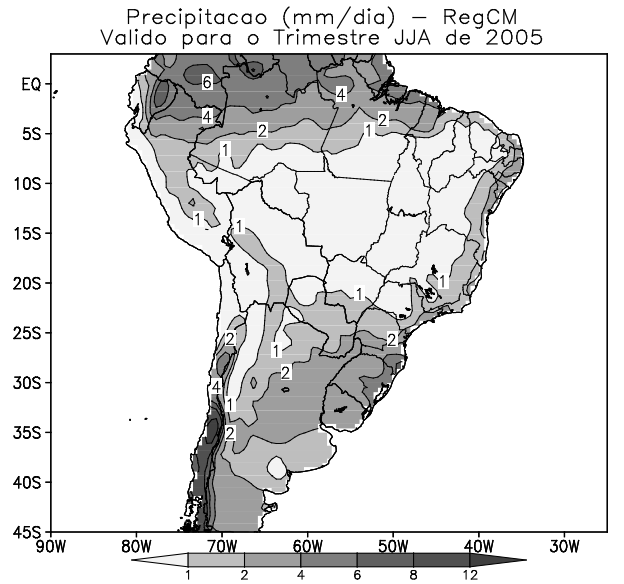

b)

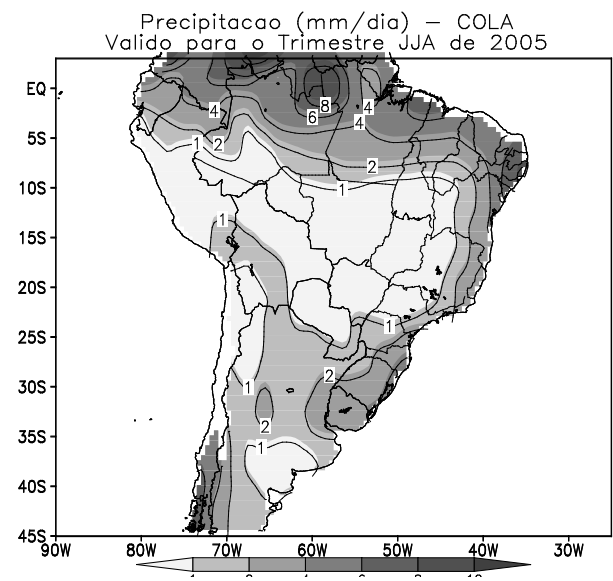

c)
2003). Esta distribuição espacial da precipitação é semelhante à da análise do CPC para o trimestre DJF/2005-2006 (Figura 2d), com maior taxa de precipitação sobre a Amazônia (até $8 \mathrm{~mm} /$

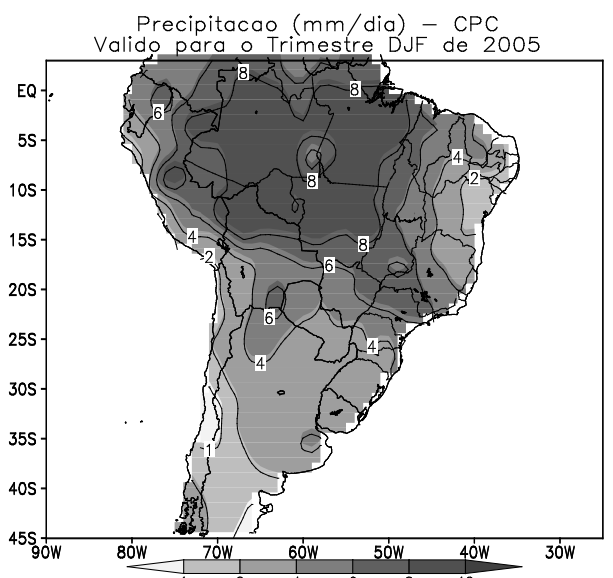

d)

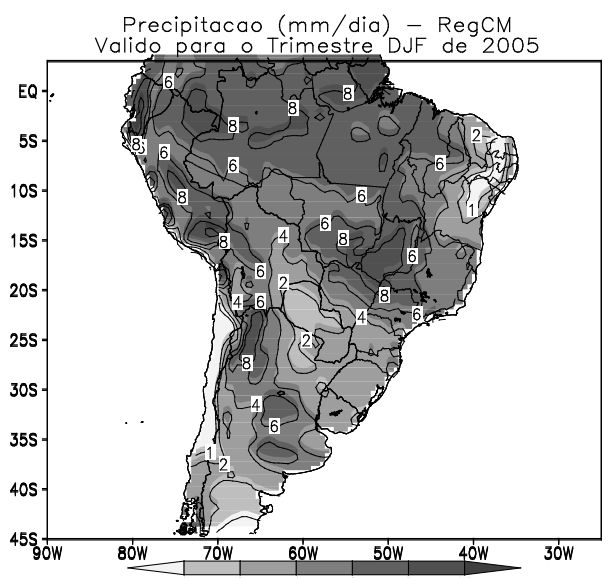

e)

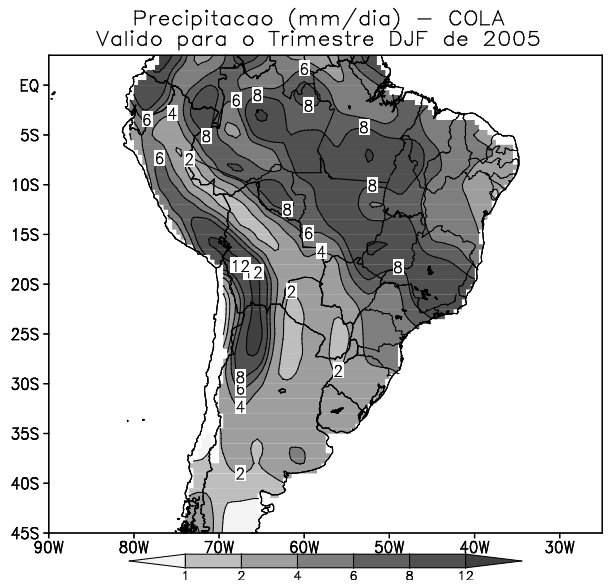

f)

Figura 2 - Precipitação média (mm/dia) para o trimestre JJA de 2005: (a) análise do CPC, (b) previsão do RegCM3 e (c) CPTEC/COLA; e para o trimestre DJF de 2005/2006: (d) análise do CPC, previsão do (e) RegCM3 e (f) CPTEC/COLA. 
dia), e parte do sudeste do Brasil (acima de $6 \mathrm{~mm} / \mathrm{dia}$ ). Chuvas de menor intensidade foram registradas no sul e nordeste do Brasil e centro-norte da Argentina (Figura 2d). A previsão do RegCM3 (Figura 2e) apresenta baixos índices de precipitação sobre o nordeste do Brasil, semelhante à análise do CPC e também ao esperado da climatologia para esta região. Também o posicionamento e intensidade das áreas, com chuva no sul $\mathrm{e}$ sudeste previsto pelo RegCM3 (Figura 2e), são mais próximos da análise do CPC (Figura 2d) do que obtidos do CPTEC/COLA (Figura 2f). Por outro lado, o RegCM3 subestima (superestima) a intensidade da chuva sobre a bacia Amazônica (centro da Argentina). Contornando o leste dos Andes, o CPTEC/COLA (Figura 2f) previu uma extensa banda de chuva seguida de outra sem chuva, o que não é observado no CPC (Figura 2d) e este erro de previsão é parcialmente corrigido na previsão do RegCM3 (Figura 2e).

As análises e previsões de temperatura do ar são apresentadas na Figura 3 para JJA/2005 e DJF/2005-2006. Para JJA/2005, o CPTEC/COLA (Figura 3c) prevê dois máximos distintos de temperatura sobre as regiões norte do nordeste do Brasil, enquanto o RegCM3 (Figura 3b) prevê temperaturas máximas mais homogêneas e semelhante ao NCEP (Figura 3a). Durante DJF/2005-2006 temperaturas mais altas foram previstas sobre o Paraguai e norte da Argentina pelo RegCM3 (Figura 3e), enquanto na análise do NCEP (Figura 3d) e CPTEC/COLA (Figura 3f) esta área mais quente ocupa uma faixa mais estreita próximo aos Andes. É aparente também que o RegCM3 subestima a temperatura do ar do NCEP sobre a bacia Amazônica, indicando um feedback positivo entre aumento da chuva e diminuição da temperatura do ar (Martinez et al., 2006; da Rocha et al., 2009).

Tanto no inverno quanto no verão, nota-se que enquanto o RegCM3 (Figura. 3b-e) tende a subestimar, o CPTEC/COLA (Figura. 3c-f) superestima a temperatura do ar da análise do NCEP (Figura 3a-d) nas áreas com temperaturas maiores na América do Sul, principalmente na planície Amazônica.

Estas avaliações subjetivas indicam que as previsões do RegCM3 corrigem alguns erros de previsão do CPTEC/COLA, principalmente o padrão de distribuição espacial de precipitação sazonal sobre o norte, nordeste e sul do Brasil.

\subsection{Impacto da regionalização: previsões do RegCM3 e CPTEC/COLA}

Foram construídas séries temporais das médias trimestrais de chuva e temperatura do ar para os 27 trimestres de previsões do CPTEC/COLA e RegCM3 nos subdomínios NDE, SDE e SUL (Figura 1). Nestes subdomínios calculou-se também o coeficiente de eficiência (E, Equação 1), correlação temporal, rmse (raiz quadrada do erro médio quadrático) e erro médio. Além destes índices, calculou-se o coeficiente de eficiência removendo o erro médio das previsões que será referido como $\mathrm{E}_{\mathrm{b}}$. Ressalta-se que os dados de temperatura do ar do CPTEC/ COLA, para o trimestre agosto-setembro-outubro de 2006, não foram considerados nas avaliações, pois não estão disponíveis.

$\mathrm{Na}$ área NDE a chuva média trimestral prevista pelo CPTEC/COLA é sempre maior que a da análise do CPC (exceto em FMA/2006) com maiores superestimativas entre os trimestres AMJ e JAS (Figura 4a). A Figura 2c ilustra que esta superestimativa estaria associada ao posicionamento errôneo da ZCIT na previsão do CPTEC/COLA, com conseqüente excesso de chuva no leste do NDE. Como ilustra a Figura 4a, as previsões do RegCM3 corrigem, pelo menos em parte, este erro sistemático do CPTEC/COLA, embora subestime a chuva da análise do CPC durante a estação chuvosa de 2006 (FMA e MAM). Esta melhoria na previsão de chuva pelo RegCM3 reflete-se no maior coeficiente de correlação linear $(0,84$ contra 0,64 do CPTEC/COLA) e no menor erro médio $(0,26$ contra $2,62 \mathrm{~mm} /$ dia do CPTEC/COLA) como sintetiza a Tabela 2. Além disto, as previsões do RegCM3 (CPTEC/COLA) fornecem coeficiente de eficiência positivo (negativo) indicando que o RegCM3 é melhor (pior) previsor da chuva trimestral do que a média observada da análise do CPC no período (Tabela 2).

No NCEP (Figura 4b) a temperatura do ar média trimestral no NDE é superior a $24^{\circ} \mathrm{C} \mathrm{em}$ todos os trimestres, com valores máximos (mínimos) durante as estações secas (úmidas). Isso indica que a chuva e a temperatura do ar estão fortemente associadas, uma vez que a disponibilidade de radiação solar nesta região, localizada próxima ao equador, é praticamente constante durante todo o ano. Então, quando a precipitação aumenta, a nebulosidade também aumenta, reduzindo a incidência de radiação solar e, por conseguinte, diminuindo a temperatura na estação chuvosa. $\mathrm{O}$ oposto também seria coerente, quando em períodos de menor precipitação a maior incidência de radiação solar aumentaria a temperatura do ar.

A Figura 4b e o coeficiente de correlação linear (Tabela 3) revelam que o RegCM3 mostra desempenho superior ao CPTEC/COLA em reproduzir a variabilidade inter-sazonal da temperatura do ar no NDE, embora subestime a temperatura da re-análise do NCEP sistematicamente em aproximadamente $1,7^{\circ} \mathrm{C}$. A justificativa para correlação de 0,89 para o RegCM3 contra 0,30 do CPTEC/COLA, deve-se aos valores trimestrais previstos pelo CPTEC/COLA estarem fora de fase em relação aos da re-análise do NCEP (Figura 4b). No CPTEC/COLA os máximos (mínimos) ocorrem nos trimestres em que o NCEP está mostrando decréscimo (aumento) da temperatura, provavelmente devido ao aumento da chuva no CPTEC/COLA (Figura 4a) não estar contribuindo para diminuir a temperatura como ocorre no NCEP (Figura 4b). Por outro lado, o bias frio do RegCM3 implica em coeficiente de eficiência negativo, o que indicaria que o RegCM3 é pior previsor de temperatura 


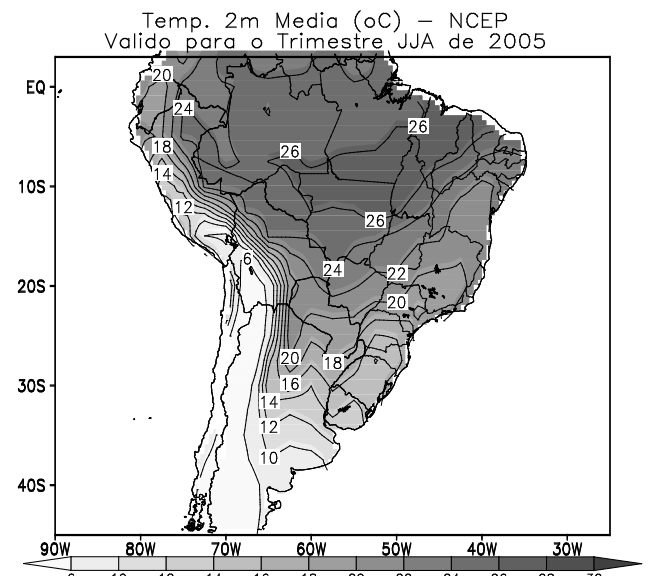

a)

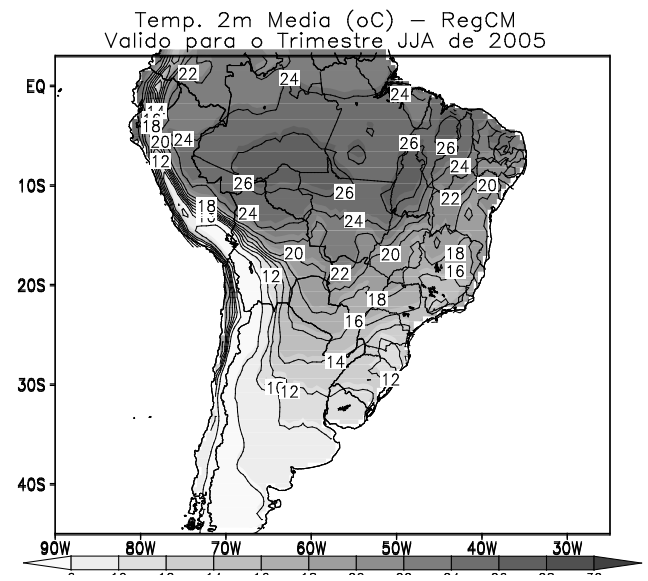

b)

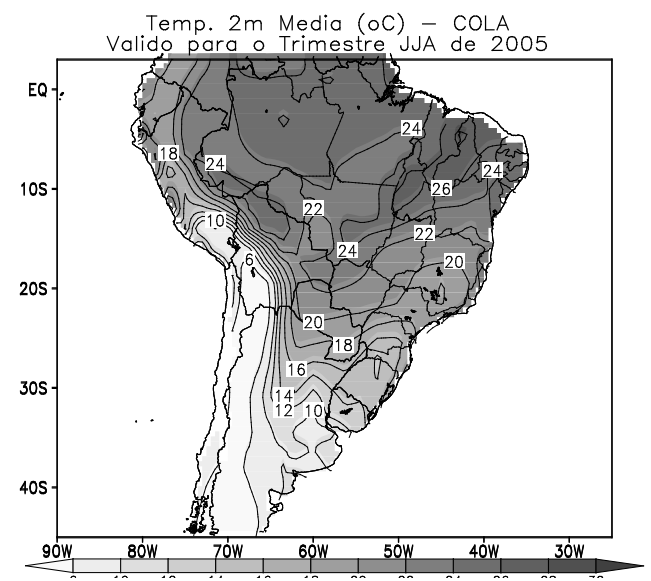

c)

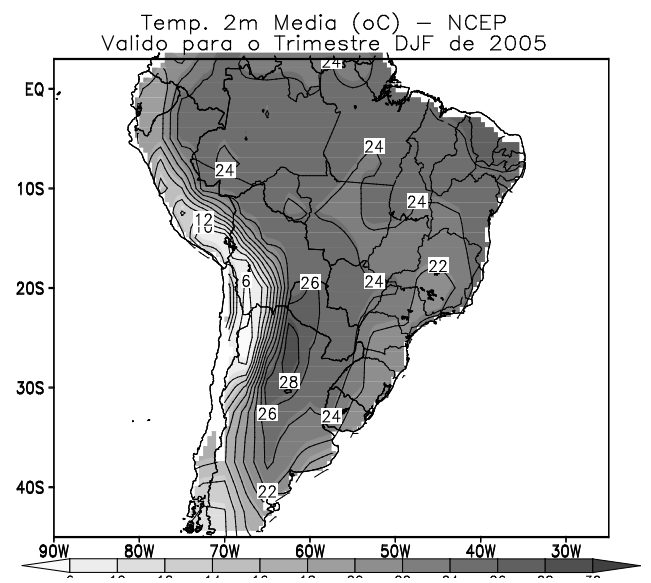

d)

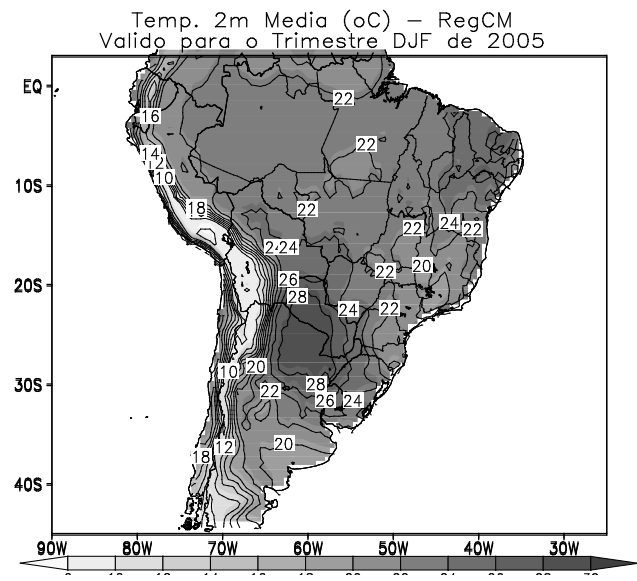

e)

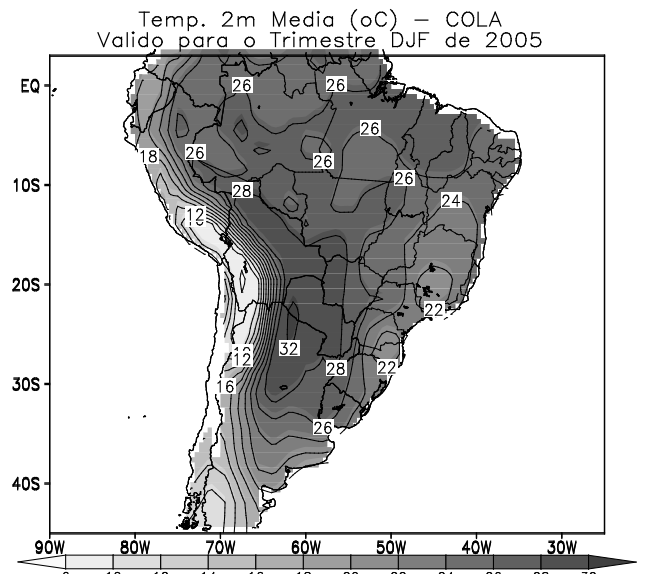

f)

Figura 3 - Precipitação média (mm/dia) para o trimestre JJA de 2005: (a) análise do CPC, (b) previsão do RegCM3 e (c) CPTEC/COLA; e para o trimestre DJF de 2005/2006: (d) análise do CPC, previsão do (e) RegCM3 e (f) CPTEC/COLA. 
do ar do que a média da análise. No entanto, apoiado na alta correlação linear do RegCM3, calculou-se o coeficiente de eficiência após a remoção do bias $\left(\mathrm{E}_{\mathrm{b}}\right)$ de ambos os modelos, e neste caso nota-se que o valor de $\mathrm{E}_{\mathrm{b}}$ é positivo e alto $(0,81)$ para o RegCM3 e se mantém negativo $(-0,03)$ para o CPTEC/

a)

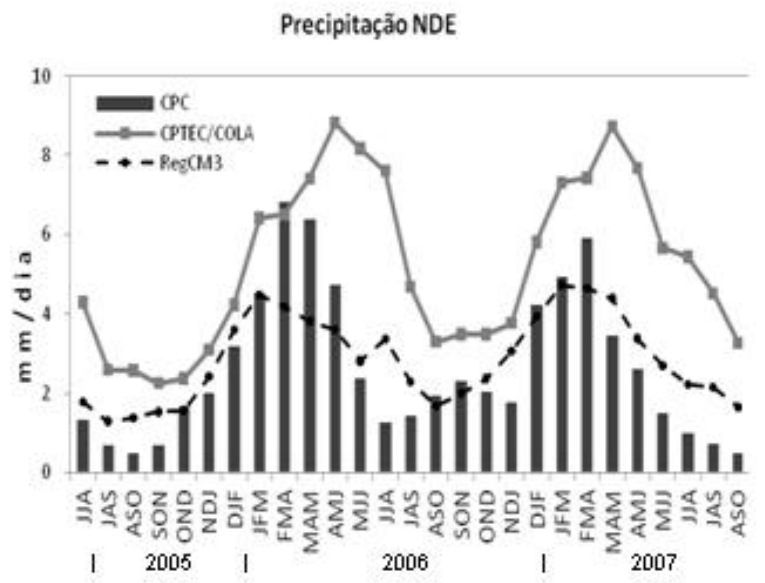

COLA. Portanto, após remover o bias sistemático o RegCM3 torna-se melhor preditor da temperatura do ar no NDE do que a média da temperatura do NCEP para todo o período.

Nota-se no subdomínio SDE (Figura 5a) que a variabilidade da chuva do CPC entre as estações do ano é

b)

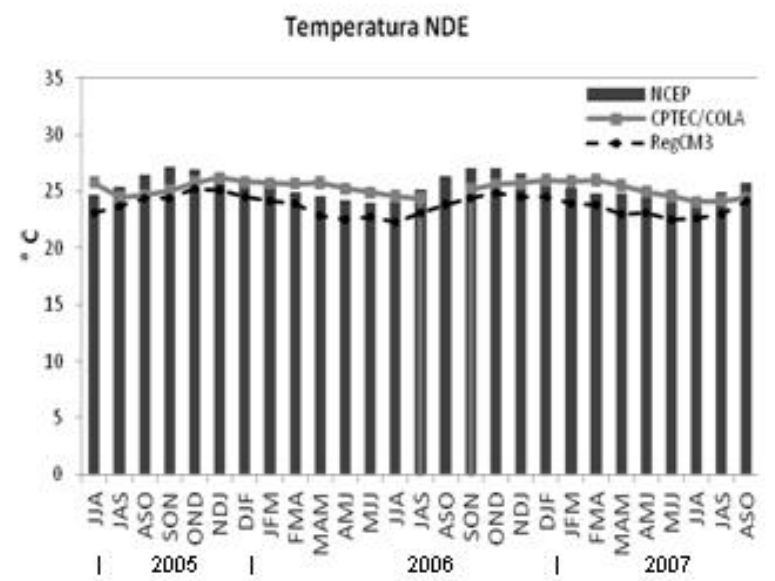

Figura 4 - Médias trimestrais da precipitação (a) da análise do CPC e temperatura do ar (b) da re-análise do NCEP e das previsões do RegCM3 e do CPTEC/COLA para a área Nordeste - NDE.

Tabela 2- Índices estatísticos (erro médio (bias), rmse, correlação temporal e coeficiente de eficiência E) e escore (ES) obtidos dos 27 trimestres de previsão de chuva dos modelos RegCM3 e CPTEC/COLA para os subdomínios NDE, SDE e SUL. Unidade do bias e rmse: mm/dia

\begin{tabular}{|c|c|c|c|c|c|c|c|c|c|c|c|c|}
\hline & \multicolumn{4}{|c|}{ NDE } & \multicolumn{4}{|c|}{ SDE } & \multicolumn{4}{|c|}{ SUL } \\
\hline & RegCM3 & ES & $\begin{array}{l}\text { CPTEC } \\
\text { /COLA }\end{array}$ & ES & RegCM3 & ES & $\begin{array}{l}\text { CPTEC/ } \\
\text { COLA }\end{array}$ & ES & RegCM3 & ES & $\begin{array}{l}\text { CPTEC/ } \\
\text { COLA }\end{array}$ & ES \\
\hline Bias & 0,26 & 1 & 2,62 & 0 & $-0,32$ & 1 & 0,66 & 0 & $-0,48$ & 1 & $-0,84$ & 0 \\
\hline rmse & 1,13 & 1 & 3,12 & 0 & 1,10 & 1 & 1,13 & 1 & 1,01 & 1 & 1,21 & 0 \\
\hline $\mathrm{r}$ & 0,84 & 2 & 0,64 & 0 & 0,91 & 2 & 0,92 & 2 & 0,63 & 2 & 0,64 & 2 \\
\hline $\mathrm{E}$ & 0,62 & 2 & $-1,86$ & 0 & 0,75 & 2 & 0,74 & 2 & 0,13 & 2 & $-0,26$ & 0 \\
\hline $\begin{array}{c}\text { ES } \\
\text { Final }\end{array}$ & & 6 & & $\mathbf{0}$ & & 6 & & 5 & & 6 & & 2 \\
\hline
\end{tabular}

Tabela 3- Idem a Tabela 2, mas para temperatura do ar $\left(\mathrm{em}^{\circ} \mathrm{C}\right)$, onde a análise para validação é a do NCEP. O valor entre parênteses indica o Eb.

\begin{tabular}{ccccccccccccc}
\hline & \multicolumn{3}{c}{ NDE } & \multicolumn{1}{c}{ SDE } & \multicolumn{3}{c}{ SUL } \\
\hline & RegCM3 & ES & $\begin{array}{c}\text { CPTEC/ } \\
\text { COLA }\end{array}$ & ES & RegCM3 & ES & $\begin{array}{c}\text { CPTEC/ } \\
\text { COLA }\end{array}$ & ES & RegCM3 & ES & $\begin{array}{c}\text { CPTEC/ } \\
\text { COLA }\end{array}$ & ES \\
\hline Bias & $-1,67$ & 0 & $-0,15$ & 1 & $-1,22$ & 0 & 0,43 & 1 & $-0,25$ & 1 & 1,24 & 0 \\
rmse & 1,72 & 0 & 1,00 & 1 & 1,51 & 0 & 0,94 & 1 & 1,18 & 1 & 1,64 & 0 \\
r & 0,89 & 2 & 0,30 & 0 & 0,85 & 2 & 0,89 & 2 & 0,94 & 2 & 0,95 & 2 \\
E & $-2,14$ & 2 & $-0,05$ & 0 & $-0,10$ & 2 & 0,57 & 2 & 0,86 & 2 & 0,74 & 2 \\
ES & $(0,81)$ & & $(-0,03)$ & & $(0,62)$ & & $(0,66)$ & & $(0,87)$ & & $(0,89)$ & \\
final & & $\mathbf{4}$ & & $\mathbf{2}$ & & $\mathbf{4}$ & & $\mathbf{6}$ & & $\mathbf{6}$ & \\
\hline
\end{tabular}


bem prevista por ambos os modelos, e isto é confirmado pelos altos valores de correlação temporal na Tabela $2(0,91$ para o RegCM3 e 0,92 para o CPTEC/COLA). Durante os períodos mais secos do ano a chuva média trimestral prevista pelo RegCM3 no SDE é mais próxima da análise do CPC do que a prevista pelo CPTEC/COLA. Verifica-se também, que no período chuvoso de 2005/2006, o CPTEC/COLA superestimou a precipitação da análise do CPC em até 1,6 mm/dia (DJF de 2005/2006). Entretanto, na segunda estação chuvosa da série temporal (Figura 5a) o RegCM3 subestimou a chuva trimestral em até 3,6 mm/dia (NDJ de 2006), enquanto o CPTEC/COLA aproximou-se mais da análise do CPC. Ainda assim, nos 27 trimestres avaliados, o erro médio do CPTEC/COLA (+0,66 $\mathrm{mm} /$ dia) é maior do que o do RegCM3 (-0,32 mm/dia). Os coeficientes de eficiência são positivos (Tabela 2) indicando que ambos os modelos são melhores previsores da chuva trimestral do que a média da análise do CPC no período. Além disto, a atribuição de escores aos índices estatísticos indica que o RegCM3 é superior ao CPTEC/COLA em prever a chuva média trimestral no SDE.

Ainda para o subdomínio SDE (Figura 5b), ambos os modelos previram corretamente a variabilidade inter-sazonal da temperatura do ar da re-análise do NCEP com correlação linear superior a 0,80 (Tabela 3). Nesta região, comparado ao NCEP o RegCM3 apresenta um erro médio frio na maioria dos trimestres, exceto em MAM e AMJ de 2006 e NDJ de 2006/2007, com as maiores subestimativas entre o inverno (JJA) e fim da primavera (OND). Após remover o bias frio das previsões trimestrais dos modelos, nota-se que o E do RegCM3 que anteriormente era negativo $(-0,10)$ torna-se positivo $(0,62)$ e próximo ao do CPTEC/COLA $(0,66)$. Mas no SDE como o RegCM3 possui maior erro médio e rmse, na soma dos escores o CPTEC/COLA apresenta-se como melhor previsor de temperatura do ar do que o RegCM3 (Tabela 3).

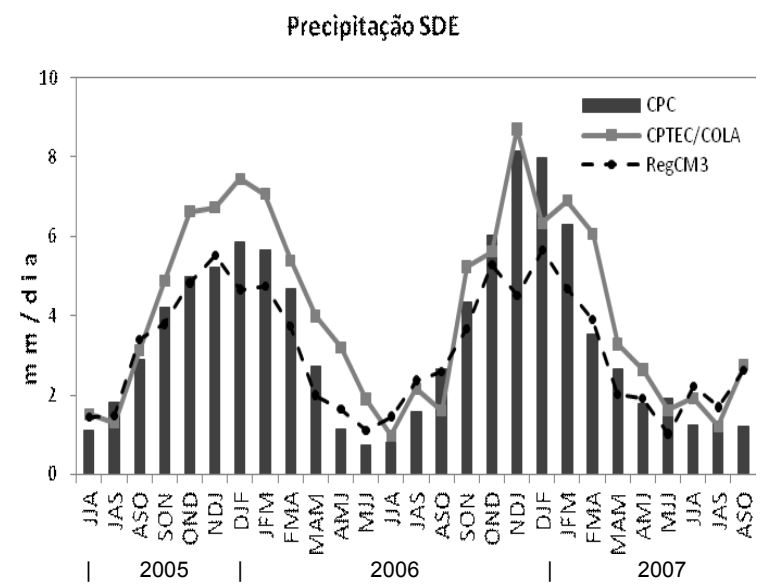

De maneira geral, a precipitação média trimestral, prevista pelo RegCM3 e CPTEC/COLA na área SUL, apresenta valores muito próximos aos da análise do $\mathrm{CPC}$ até $\mathrm{SON}$ de 2006 (Figura 6a). Entre os trimestres DJF de 2006/2007 e MJJ de 2007, ambos os modelos subestimam a chuva do CPC em até $30 \%$, com a pior previsão do RegCM3 ocorrendo em FMA de 2007. Essa subestimativa repercute no coeficiente de eficiência negativo para o CPTEC/COLA (-0,26 na Tabela 2). Nesta área o RegCM3, com coeficiente de eficiência positivo $(0,13)$, menores erro médio e rmse, tem melhor desempenho em prever a chuva trimestral se comparado a média da análise do CPC e ao CPTEC/COLA (Tabela 2).

A exemplo da região SDE, na SUL a variabilidade da temperatura do ar entre as estações também é corretamente prevista pelos modelos, com o RegCM3 reduzindo o erro médio quente do CPTEC/COLA, e aproximando-se mais da re-análise do NCEP (Figura 6 b e Tabela 3). A correta previsão do ciclo anual implica em coeficientes de correlação superiores a 0,93 para os dois modelos. No entanto, a temperatura do ar prevista pelo RegCM3 é mais próxima da re-análise do NCEP, do que a do CPTEC/COLA na maioria dos trimestres (Figura $6 b)$, refletindo-se em maior coeficiente de eficiência para as previsões do RegCM3 (Tabela 3). Na soma dos escores, o RegCM3 apresenta-se como melhor previsor do que o CPTEC/ COLA para temperatura do ar média trimestral no SUL. 3.3

\subsection{Previsões do RegCM3 versus climatologia}

Além de comparar as previsões dos modelos, é importante entender se a regionalização "adiciona valor", quando as previsões do RegCM3 são comparadas com a climatologia observada em cada trimestre. Ou seja, se as previsões superaram a utilização dos valores climatológicos observados como

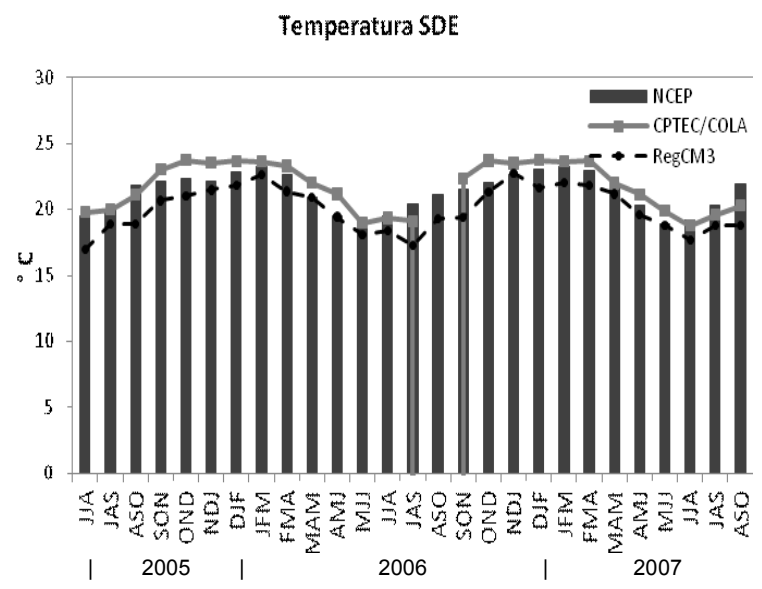

Figura 5 - Idem à Figura 4, mas para o subdomínio SDE. 
preditor trimestral. Então, realizou-se uma avaliação objetiva entre as previsões do RegCM3 e a climatologia da chuva e temperatura do ar das análises do CPC e NCEP, respectivamente, através do coeficiente de eficiência E' (Equação 2).

Para o subdomínio NDE tanto a climatologia quanto o RegCM3 não reproduziram os máximos de precipitação trimestral da análise do CPC durante o período chuvoso de 2006 (Figura 7a). Já para os períodos secos de 2006 e 2007, a climatologia aproxima-se mais da análise do CPC, do que as previsões do RegCM3. Ainda assim, considerando todo o período de avaliação, no NDE o coeficiente de eficiência E' positivo (0,37 na Tabela 4) indica o RegCM3 como melhor previsor da chuva média trimestral do que a climatologia. Além disso, o erro médio no período é menor utilizando o RegCM3, uma vez que superestima a precipitação em $0,3 \mathrm{~mm} /$ dia, enquanto a climatologia subestima em $0,6 \mathrm{~mm} /$ dia (Tabela 4 ).

A variabilidade temporal das médias trimestrais de temperatura do ar da re-análise do NCEP é corretamente representada pela previsão do RegCM3 e pela climatologia no NDE (Figura 7b). No entanto, nota-se que a climatologia apresenta um erro médio frio $\left(-0,4^{\circ} \mathrm{C}\right)$, que é menor do que no RegCM3 $\left(-1,7^{\circ} \mathrm{C}\right.$ na Tabela 4$)$. Associado a este erro médio frio o coeficiente de eficiência E' das previsões do RegCM3 é negativo (-1,00 na Tabela 4). No entanto, removendo o erro médio das previsões trimestrais, o E' ${ }_{\mathrm{b}}$ torna-se positivo $(0,78)$, revelando assim o RegCM3 como melhor preditor da temperatura do ar no NDE do que a climatologia.

De maneira geral, durante os períodos secos no subdomínio SDE, a chuva climatológica e prevista pelo RegCM3 aproxima-se mais da análise do CPC do que nos períodos chuvosos (Figura 8a). Comparativamente, no período chuvoso (DJF, JFM e FMA) de 2005/2006, enquanto a previsão do RegCM3 mostra forte subestimativa da chuva, os valores climatológicos são mais próximos do CPC. Na estação chuvosa seguinte, NDJ e DJF de 2006/2007, a elevada taxa de precipitação do CPC esteve associada ao maior número de sistemas frontais, ZCAS e vórtices ciclônicos em altos níveis, que provocaram altos índices de chuvas diárias como as registradas em Votuporanga-SP $(162,6 \mathrm{~mm}$ no dia $02 \mathrm{de}$ janeiro), Ribeirão Preto-SP (95 mm no dia 03 de janeiro),
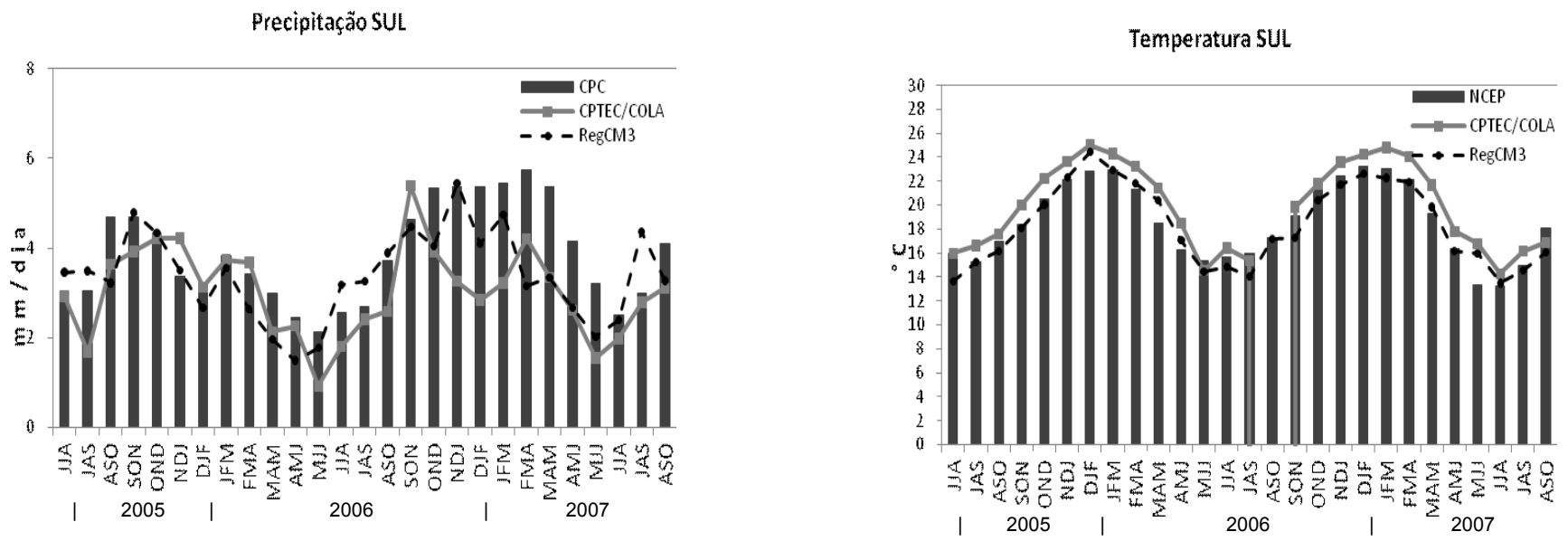

Figura 6 - Idem à Figura 4, mas para o subdomínio SUL.

Tabela 4- Médias e coeficientes de eficiência (E' e E’b) da precipitação e temperatura do ar para os três subdomínios de estudo.

\begin{tabular}{|c|c|c|c|c|c|c|c|c|c|}
\hline \multirow[b]{3}{*}{ Área } & \multicolumn{4}{|c|}{ Precipitação } & \multicolumn{4}{|c|}{ Temperatura } & \multirow{3}{*}{$E_{b}^{\prime}$} \\
\hline & \multicolumn{3}{|c|}{ Média (mm/dia) } & \multirow[t]{2}{*}{$E^{\prime}$} & \multicolumn{3}{|c|}{ Média $\left({ }^{\circ} \mathrm{C}\right)$} & $E^{\prime}$ & \\
\hline & $\mathrm{CPC}$ & RegCM3 & Climatológica & & NCEP & RegCM3 & Climatológica & & \\
\hline $\mathrm{NDE}$ & 2,6 & 2,9 & 2 & 0,37 & 25,4 & 23,7 & 25 & -1 & 0,78 \\
\hline $\mathrm{SDE}$ & 3,4 & 3,1 & 3,5 & 0,57 & 21,3 & 20 & 20,3 & 0,15 & - \\
\hline SUL & 3,9 & 3,4 & 4,3 & 0,23 & 18,6 & 18,3 & 18,2 & 0,68 & - \\
\hline
\end{tabular}


Precipitaçădo NDE

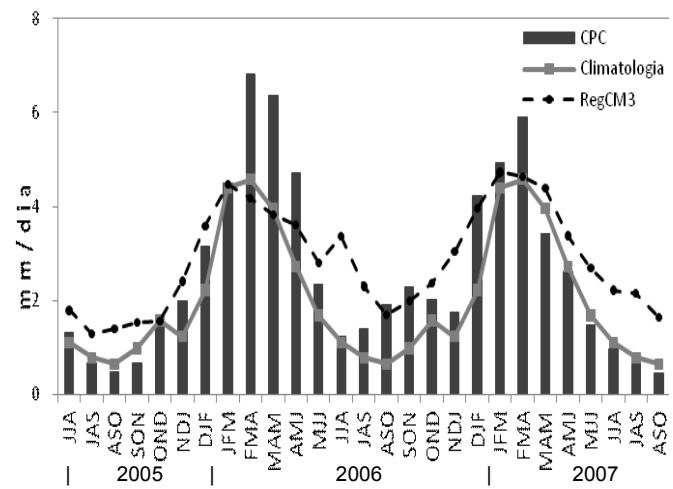

Temperatura NDE

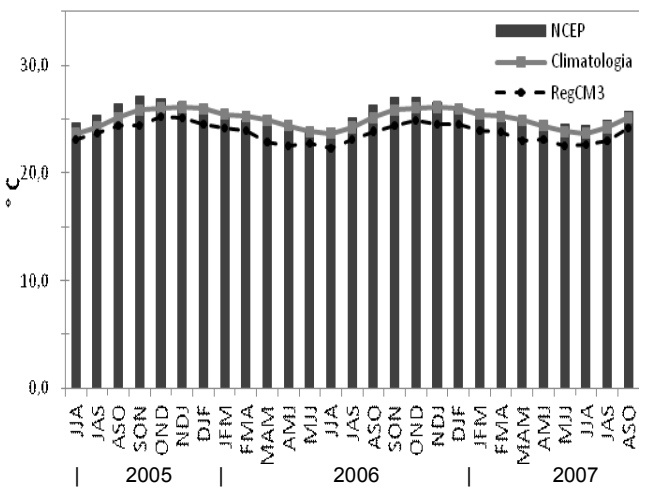

Figura 7 - Médias trimestrais da precipitação (a) da análise do CPC e temperatura do ar (b) da re-análise do NCEP e das previsões do RegCM3 e climatologia para a área do Nordeste - NDE.
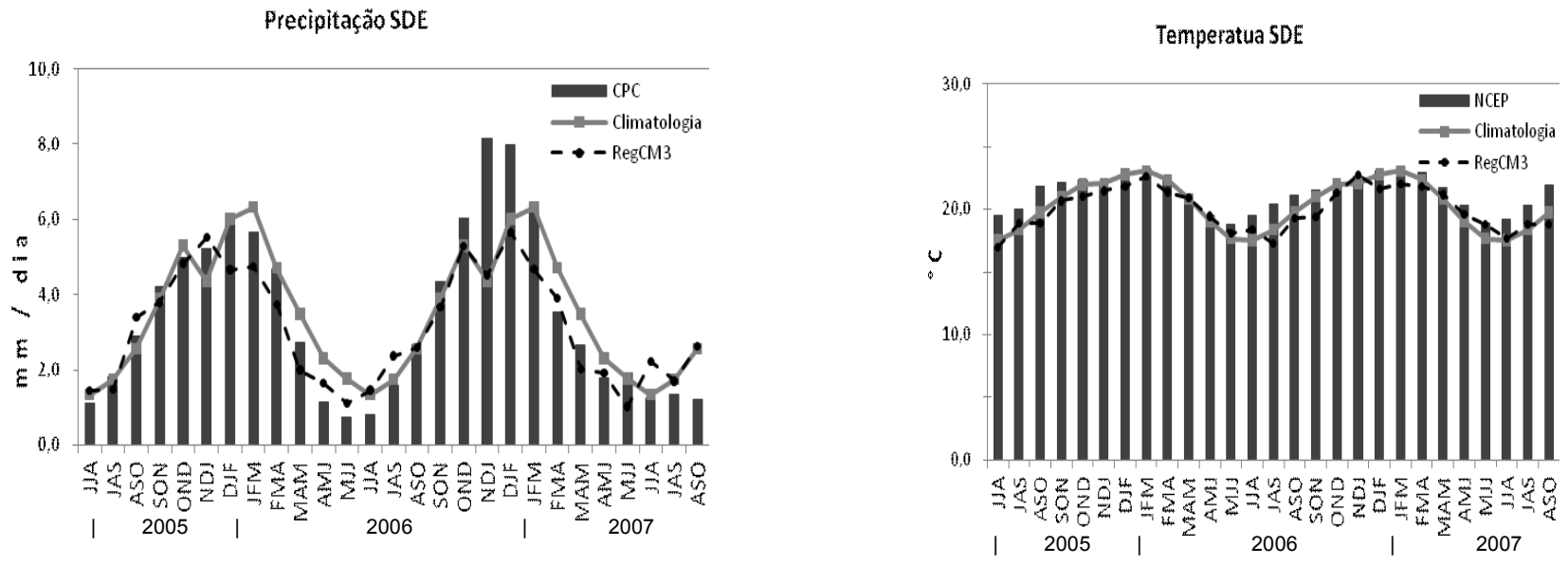

Figura 8 - Idem à Figura 7, mas para o subdomínio SDE.

Tabela 5- Diferença relativa $(\%)$ da chuva trimestral e diferença absoluta da temperatura do ar $\left({ }^{\circ} \mathrm{C}\right)$ de cada uma das 4 previsões em relação à iniciada no dia 16.

\begin{tabular}{ccccccc}
\hline & \multicolumn{2}{c}{ Diferença relativa da chuva (\%) } & \multicolumn{2}{c}{ Diferença absoluta da temperatura do ar $\left({ }^{\circ} \mathbf{C}\right)$} \\
\hline $\begin{array}{c}\text { Data inicial } \\
\text { da previsão }\end{array}$ & NDE & SDE & SUL & NDE & SDE & SUL \\
\hline 16 & 0,00 & 0,00 & 0,00 & 0,00 & 0,00 & 0,00 \\
17 & 0,00 & $-9,06$ & 0,00 & 0,09 & $-0,05$ & $-0,15$ \\
18 & $-1,25$ & 0,00 & 1,39 & 0,02 & $-0,04$ & $-0,18$ \\
19 & 2,00 & $-6,00$ & 1,85 & $-0,10$ & $-0,02$ & $-0,04$ \\
20 & $-3,00$ & $-4,00$ & 1,85 & $-0,28$ & -0.03 & 0,13 \\
\hline
\end{tabular}


Cravinhos-SP (120 mm no dia 14 de janeiro) e São Paulo-SP (103,3 mm no dia 8 de fevereiro) conforme o Climanálise $(2007 a, b)$. Esses eventos de chuva intensa no SDE, que elevaram a média trimestral do $\mathrm{CPC}$, não foram previstos pelo RegCM3. Ainda assim, quando todo período é considerado o E' de 0,57 (Tabela 4) indica que o RegCM3 é melhor preditor da chuva no subdomínio SDE do que a climatologia.

Assim como para o NDE, a climatologia de temperatura do ar no SDE (Figura 8b) diminui o erro médio frio das previsões do RegCM3 em 15 dos 27 trimestres avaliados. No entanto, a Tabela 4 indica que as previsões do RegCM3 fornecem coeficiente de eficiência positivo para chuva e para temperatura do ar, mostrando que no SDE, o RegCM3 é melhor preditor dos valores médios trimestrais do que a climatologia.

$\mathrm{Na}$ área SUL (Figura 9a) entre os trimestres NDJ de 2005/2006 à MJJ de 2006 as médias trimestrais do CPC foram inferiores à média climatológica. Isto pode ser explicado pela menor freqüência de sistemas frontais e pela rápida passagem dos mesmos, o que desfavoreceu a ocorrência de chuva nesta região (Climanálise, 2006a,b,c). No mesmo período, o RegCM3 previu valores trimestrais de chuva mais próximos do CPC do que a climatologia, enquanto o oposto ocorre entre FMA e MJJ de 2007 (Figura 9a). Considerando todo o período de análise, o erro médio das previsões do RegCM3 é menor do que o climatológico, e o coeficiente de eficiência E' positivo (0,23 na Tabela 4) indica que as previsões do RegCM3 de chuva média trimestral superam a climatologia.

Para a temperatura do ar, tanto a climatologia quanto o RegCM3 representam corretamente a evolução temporal dos valores médios trimestrais da re-análise do NCEP no subdomínio SUL (Figura 9b). O elevado valor do coeficiente de eficiência E' $(0,68$ na Tabela 4$)$, o maior entre as três regiões avaliadas, indica o correto desempenho do RegCM3 na previsão da fase e amplitude da temperatura do ar no SUL, superando a climatologia trimestral.

Conforme discutido e sintetizado na Tabela 4, nos três subdomínios considerados é possível afirmar que o RegCM3 apresenta-se como melhor preditor de precipitação e temperatura do ar médias trimestrais do que a climatologia.

\subsection{Conjunto de Previsões}

Uma questão importante a ser discutida é que nas avaliações anteriores considerou-se apenas uma realização com o RegCM3. No entanto, as previsões climáticas mostram grande sensibilidade a pequenas perturbações nas condições iniciais, parametrizações físicas utilizadas nos modelos numéricos e forçantes como a temperatura da superfície do mar (Chervin e Schneider, 1976; Rowell, 1998). Em um cenário ideal, várias previsões regionais devem ser realizadas partindo de diferentes previsões do modelo global. No entanto, para o período considerado só estava disponível um membro do GCM CPTEC/COLA, inviabilizando esta metodologia. Como alternativa para discutir os erros associados à utilização de apenas uma previsão, normalmente referida como determinística, além da previsão iniciada no dia 16 (Controle) foram realizadas mais 4 previsões iniciando nos dias 17, 18, 19 e 20. Em função da disponibilidade computacional esta metodologia foi aplicada apenas à previsão de janeiro de 2006.

A Tabela 5 apresenta a diferença relativa (absoluta para temperatura do ar) da precipitação média trimestral para cada uma das previsões, onde as diferenças foram calculadas sempre em relação à previsão iniciada no dia 16 (Controle). As diferenças relativas da chuva média trimestral são sempre pequenas e inferiores à $\pm 10 \%$ (Tabela 5), e não se nota redução do erro de previsão ao se aproximar do trimestre de interesse, ou seja, reduzindo o tempo de "spin-up". Resultado semelhante é obtido para a temperatura do ar (Tabela 5), com diferenças absolutas em relação ao Controle não excedendo $\pm 0,3^{\circ} \mathrm{C}$. Estes resultados
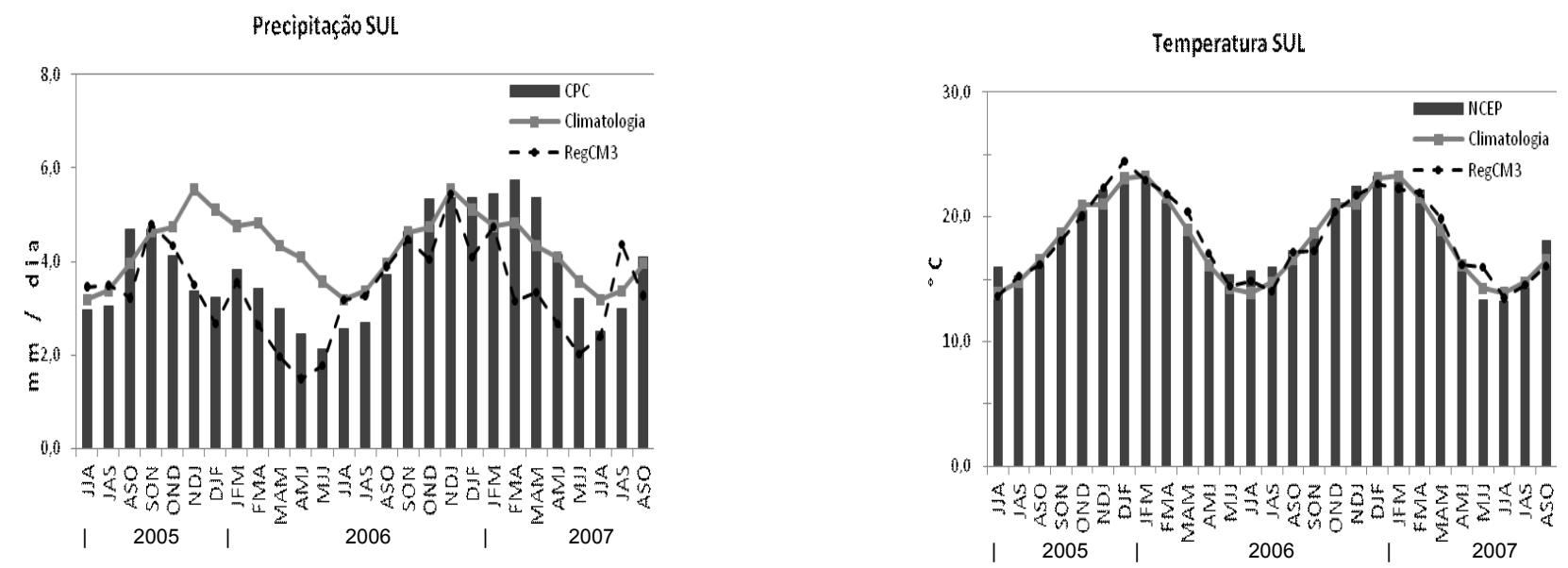

Figura 9 - Idem à Figura 7, mas para o subdomínio SUL. 
indicam que outra metodologia deve ser buscada para gerar um conjunto de previsões regionais para avaliação probabilística. Uma delas seria utilizar um conjunto de previsões do CPTEC/ COLA para realizar um conjunto de previsões sazonais com o RegCM3. Esta possibilidade será explorada futuramente em conjunto com pesquisadores do CPTEC através de previsões de períodos passados (hindcast).

\section{SUMÁRIO E CONCLUSÕES}

Este trabalho analisou o impacto da regionalização de previsões climáticas sobre o Brasil utilizando o modelo climático regional RegCM3 (Pal et al. 2007) aninhado ao GCM CPTEC/COLA (Bonatti, 1996). As 27 previsões (de chuva e temperatura do ar) avaliadas foram iniciadas às 00:00 UTC do dia 16 de cada mês, e prosseguiram até o último dia dos 3 meses seguintes. Para avaliação objetiva, os primeiros 14 (ou 15 dependendo se o mês em questão possui 30 ou 31 dias) foram desconsiderados e calcularam-se médias trimestrais dos três meses seguintes ao mês inicial.

Comparações das previsões do RegCM3 e CPTEC/ COLA com observações mostraram que alguns erros sistemáticos do CPTEC/COLA são corrigidos pelo RegCM3. Por exemplo, o RegCM3 corrige o incorreto posicionamento da área de chuva associada à ZCIT no CPTEC/COLA, que desloca no tempo a estação chuvosa do nordeste do Brasil para MAM o que contradiz observações que a identificam em FMA (Marengo et al., 2003; Misra, 2006). Associado a este deslocamento, as previsões do CPTEC/COLA superestimam a chuva trimestral observada e também defasam em um/dois meses o pico da estação chuvosa no nordeste do Brasil quando comparado à observação. Ao corrigir este erro, as previsões do RegCM3 fornecem maior correlação temporal e coeficiente de eficiência positivo para chuva no NDE. Este melhor desempenho também é encontrado nas previsões de temperatura do ar, com maior correlação temporal $(0,89)$ para o RegCM3 do que CPTEC/COLA $(0,30)$, indicando maior habilidade do RegCM3 em prever a evolução temporal da temperatura no NDE. Após remover o erro sistemático frio de ambos os modelos (-1,67 do RegCM3 e -0,15 do CPTEC/COLA), o coeficiente de eficiência positivo e próximo de $1(0,81)$ indica também maior destreza das previsões do RegCM3 comparadas às do CPTEC/COLA.

Para o SDE, ambos os modelos apresentam correlação temporal acima de 0,90 para a chuva e acima de 0,84 para temperatura do ar, e coeficiente de eficiência positivo acima de 0,60. Nesta área, o CPTEC/COLA apresenta ligeira superioridade nas previsões de temperatura do ar devido ao menor erro médio e rmse. Por outro lado, as previsões de chuva do RegCM3 são ligeiramente superiores às do CPTEC/COLA devido ao menor erro médio.
Na terceira e última área avaliada, SUL, as previsões de chuva e temperatura do ar do RegCM3 possuem menor erro médio e rmse, além de coeficiente de eficiência positivo, justificando assim a superioridade do RegCM3 perante o CPTEC/COLA.

Durante os 27 trimestres, a verificação objetiva das previsões climáticas do RegCM3 em relação à climatologia, revelou que nas três áreas (NDE, SDE e SUL) o RegCM3 é mais eficiente em prever os valores médios trimestrais de chuva e a temperatura do ar do que a média climatológica observada.

Embora as análises apresentadas possuam limitações, já que utilizaram apenas uma realização de cada um dos modelos, nota-se que a regionalização com o RegCM3 adiciona informações às previsões do CPTEC/COLA, corrigindo alguns erros sistemáticos deste. Futuramente, pretende-se analisar um maior número de previsões regionais, ou utilizando várias previsões do CPTEC/COLA, ou perturbando a TSM persistida e realizando várias previsões como o RegCM3 partindo de uma previsão do CPTEC/COLA, ou seja, construindo um conjunto de previsões trimestrais e analisando os resultados probabilisticamente.

\section{AGRADECIMENTOS}

Ao CNPq pela concessão da bolsa de Mestrado ao primeiro autor, ao laboratório GREC/USP pela infra-estrutura, à FAPESP (Procs. 2001/13925-5 e 03/01271-6), à CAPES/ PROCAD, projeto EUROBRISA e FAPERGS. Ao NCEP, CPC e ICTP por disponibilizarem os dados de re-análise, análise de precipitação e código-fonte do RegCM3, respectivamente. À Dra. Cintia Uvo pelas importantes sugestões, ao Carlos Renato de Souza do Grupo de Previsão Climática do CPTEC/INPE e aos revisores anônimos que contribuíram para a melhoria deste artigo.

\section{REFERÊNCIAS BIBLIOGRÁFICAS}

ALVES, L. M; MELO, A. B. C.; CHOU, S.C. NOBRE, P. Estudo comparativo entre a precipitação sazonal simulada pelo modelo ETA e observada sobre o Brasil. In: $\mathbf{1 1}^{\circ}$ Congresso Brasileiro de Meteorologia, Rio de Janeiro, 2000. 1 CD-ROM. ALVES, J. M. B.; CAMPOS, J. N. B.; FILHO, F. A. S.; MONCUNNIL, D. F.; SILVA, E. M.; BARBOSA, W. L.; FERREIRA, A. G.; SUN, L.; MOURA, A. D. Uma avaliação preliminar de simulações climáticas de um modelo regional espectral aninhado em um modelo global (echam4.5) sobre o setor norte da região nordeste do brasil (1971-2000).

Revista. Brasileira. Meteorologia, v.20, n.2, p.40-55, 2005. BONATTI, J. P. Modelo de circulação geral atmosférico do CPTEC. EdiçãoEspecial 10 anosde Climanálise, 1996.http://climanalise. cptec.inpe.br/ rclimanl/boletim/cliesp10a/bonatti.html CHERVIN, R. M.; SCHNEIDER, S. H. A study of the 
response of NCAR GCM climatological statistics to random pertubations: Estimating noise levels. Journal of the Atmospheric Sciences, n. 33, p. 391-404, 1976.

CHOU, S. C.; NUNES; A. M. B.; CAVALCANTI, I. F. A. Extended range forecasts over South America using the regional ETA model. Journal of Geophysical Research, Washington, v.105, p. 10147-10160, 2000. CHOU, S.C.; BUSTAMANTE, J.F.; GOMES, J.L. Evaluation of Eta Model seasonal precipitation forecasts over South America. Nonlinear Processes in Geophysics, v.12, p.537555. 2005.

CLIMANÁLISE, Boletim de Monitoramento de Análise Climática. São Paulo: SCT/INPE-CPTEC, v. 21, n. 05, maio de 2006a. . Boletim de Monitoramento de Análise Climática. São Paulo: SCT/INPE-CPTEC, v. 21, n. 11, novembro de 2006 b. . Boletim de Monitoramento de Análise Climática. São Paulo: SCT/INPE-CPTEC, v. 21, n. 12, dezembro de 2006c. . Boletim de Monitoramento de Análise Climática. São Paulo: SCT/INPE-CPTEC, v. 22, n. 01, janeiro de 2007a.

. Boletim de Monitoramento de Análise Climática. São Paulo: SCT/INPE-CPTEC, v. 22, n. 02, fevereiro de 2007b.

CUADRA, Santiago Vianna; ROCHA, Rosmeri Porfírio da . Simulação Numérica do Clima de Verão Sobre o Sudeste do Brasil e sua Variabilidade. Revista Brasileira de Meteorologia, v. 21, p. 271-282, 2006.

DA ROCHA, R. P.; MORALES, C. A.; CUADRA, S. V.; AMBRIZZI, T. Precipitation diurnal cycle and summer climatology assessment over South America: An evaluation of Regional Climate Model version 3 simulations. Journal of Geophysical Research, v. 114, p. 1-19, 2009.

DICKINSON, R. E.; ERRICO, R. M.; GIORGI, F.; et al. A regional climate model for western United States. Climate Change. v.15, p.383-422, 1989.

DICKINSON, R. E.; HENDERSON-SELLERS, A.; KENNEDY, P. J. Biosphere-atmosphere-transfer scheme (BATS) version $1 \mathrm{E}$ as coupled to NCAR Community Climate Model. NCAR Technical Note, National Center for Atmosphere Research, Boulder, 1993.

FERNANDEZ, J. P. R.; FRANCHITO, S. H.; RAO ,V. B. Simulation of the summer circulation over South America by two regional climate models. Part I: Mean climatology, Theoretical and Applied Climatology, 2006a.

. Simulation of the summer circulation over South America by two regional climate models. Part II: A comparison between 1997/1998 El Nino and 1998/1999 La Nina events. Theoretical and Applied Climatology, 2006b.

FRITSCH, J. M.; CHAPPELL, C. F. Numerical prediction of convectively driven mesoscale pressure systems. Part I: Convective parameterization. Journal Atmosphere Science, v.37, p. 1722-1733, 1980.
GRELL, G.A. Prognostic evaluation of assumptions used by cumulus parameterizations. Monthly Weather Review, v. 121, p. 764-787, 1993.

GIORGI, F., MARINUCCI, M.R. Validation of a Regional Atmospheric Model over Europe: Sensitivity of wintertime and summertime simulations to selected physics parametrizations and cover boundary conditions. Quarterly Journal of the Royal Meteorological Society, 117 pp. 1171-1206, 1991. HOLTSLAG, A, D; BRUIJN, E.; PAN, H.-L. A high resolution air mass transformation model for short-range weather forecasting. Monthly Weather Review, n. 118, p. 1561-1575, 1990.

HOREL, J. D.; HAHMANN, A. N.; GEISLER, J. E. An investigation of the annual cycle of convective activity over the tropical Americas. Journal of Climate, Boston, v. 2, p. 1388 - 1403, 1989.

KALNAY, E.; et al, NCEP/NCAR 40-year Reanalysis project. Bulletin of the American Meteorological Society, v. 77, p. 437-471, 1996. KIEHL, J.T.; HACK, J.J.; BONAN, G.B.; et al. Description of the NCAR Community Climate Model (CCM3). Technical Note, NCAR/TN-420+STR, Colorado: Boulder, 1996, 152 p. KODAMA, Y. M. Large-scale common features of sub-tropical precipitation zones (the Baiu Frontal Zone, the SPCZ, and the SACZ). Part I: characteristics of subtropical frontal zones. Journal of Meteorological Society of Japan, v. 70, p. 813-835, 1992.

KOUSKY, V. E. Diurnal rainfall variation in Northeast Brazil. Monthly Weather Review, v.108, n. 4,p.488-498, abrilde 1980. LIEBMANN, B.; KILADS, G. N.; MARENGO, J. A.; et al. Submontly convecive variability over South America and South Atlantic Convergence Zone. Journal of Climate, Boston, v. 12, p. 1877-1891, 1999.

LOVELAND, T.R., REED, B.C., BROWN, J.F., OHLEN, D.O., ZHU, J, YANG, L., and MERCHANT, J.W. Development of a global land cover characteristics database and IGBP DISCover from 1-km AVHRR data. International Journal of Remote Sensing 21(6/7): 1303-1330, 2000

MARENGO, J. A; CAVALCANTI, I. F. A.; SATYAMURTY, P.; et al. Assessment of regional seasonal rainfall predictability using the CPTEC/COLA atmospheric GCM. Climate Dynamics, v. 21, p. 459-475, 2003.

MARTÍNEZ C., D. ; da ROCHA, R. P. ; BEZANILLA M. A.; ALVAREZ E. L; REYES F., J. P; SILVA V. Y.; ARRITT, R. W. Sensitivity studies of the RegCM-3 simulation of summer precipitation, temperature and local wind field in the Caribbean Region. Theoretical and Applied Climatology, v. 86, p. 5-22, 2006.

MISRA, V.; KANAMITSU, M. Anomaly Nesting: AMethodology to Downscale Seasonal Climate Simulations from AGCMs. Journal of Climate, v.17, n. 17, p.3249-3262, 2004. 
MISRA, V. Understanding the predictability of seasonal precipitation over northeast Brazil. Tellus, v . 58a, p. 307-319, 2006.

NASH J. E.; SUTCLIFFE J. V. River flow forecasting through conceptual models part I - A discussion of principles. Journal of Hydrology, v. 10, 3 ed., p. 282-290, 1970.

PAL, J.S.; ERIC, E. S. e ELFATHI, A.B.E. Simulation of regionalscale water energy budgets: Representation of subgrid cloud and precipitation process within RegCM. Journal of Geophysical Research, v.105, p.29.579-29.594, 2000.

PAL, J.S. ; GIORGI, F. ; BI, X. ; ELGUINDI, N. ; SOLMON, F. ; GAO, X. ; RAUSCHER, S.; FRANCISCO, R. ; ZAKEY, A. ; WINTER, J ; ASHFAQ, M. ; SYED, F. ; BELL, J.1.; DIFFENBAUGH, N.s. ; ROCHA, R. P. da . Regional Climate Modeling for the Developing World: The ICTP RegCM3 and RegCNET. Bulletin of the American Meteorological Society, v. 88, p. 1395-1409, 2007.

RAUSCHER, S. A., A. SETH, B. LIEBMANN, J.-H. QIAN, and S. J. CAMARGO. Regional climate model-simulated timing and character of seasonal rains in South America, Monthly Weather Review, 135, 2642-2657, 2007

REBOITA, M.; da ROCHA, R. P.; AMBRIZZI, T. Evaluation of the latent and sensible heat fluxes simulated by RegCM3 over the South Atlantic from 1990 to 1994. CLIVAR Exchanges, Southampton, United Kingdom, v. 10, n. 4, p. 58-60, 2005.

ROWELL, D. P. Assessing potential seasonal predictability with an ensemble of multidecadal GCM simulations. Journal of Climate v. 11, p. 109-120, 1998.

SEN, O.L.; WANG, Y.; WANG, B. Impact of Indochina deforestation on the East-Asian summer monsoon. Journal of Climate, v. 17, p. 1366-1380, 2004a

SEN, O.L.; WANG, Y.; WANG, B. Re-greening the desertification lands in northern China: Implications from a regional climate model experiment. Journal of Meteorological Society of Japan, n. 6, v.82 , p. 1679-1693 2004b

SETH, A.; ROJAS, M. Simulation and sensitivity in a nested modeling system for SouthAmerica. Part I: Reanalyses boundary forcing, Journal of Climate, n. 16, p. 2437-2453, 2003.

SETH, A.; RAUSCHER, S.A.; CAMARGO, S. J.; et al. RegCM3 regional climatologies for South America using reanalysis and ECHAM global model driving fields. Climate Dynamics, v. 28. p. 461-480, 2007.

SILVA, J., M. Simulação regional da variabilidade climática interanual no norte do Nordeste brasileiro, 2008. $81 \mathrm{f}$. Dissertação de Mestrado - Programa de Pós-graduação do Instituto de Astronomia, Geofísica, e Ciências Atmosféricas. Universidade de São Paulo, São Paulo, 2008.

SILVA, S. B. V., KOUSKY, E. V., SHI, W., HIGGINS, W; An Improved Grided Historical Daily Precipitation Analysis for Brazil. Journal Hydrometeorology. v. 8, p. 848-861, 2007.

SUN, L., D.V. MONCUNILL, H. Li, A. D. MOURA, F. A de SOUZA FILHO: Climate Downscaling over Nordeste, Brazil, using the NCEP RSM97 - Journal of Climate, $n$. 18, p. 551-567, 2005.

WEBER,A.; BUCKNER, M.; WEBER, J. Statistical Performance of Several Mesoscale Atmospheric Dispersion Models. Journal of Applied Meteorology, NOAA Central Library, Department of Commerce, v. 21, n. 11, p. 1633-1644, 1982. 